\title{
Adaptive fluctuation imaging captures rapid subcellular dynamics
}

\author{
Ida S. Opstad ${ }^{1, *}$, Florian Ströhl ${ }^{1}$, Åsa B. Birgisdottir ${ }^{2}$, Sebastián Maldonado ${ }^{1}$, Trine Kalstad ${ }^{2}$, \\ Truls Myrmel ${ }^{2}$, Krishna Agarwal ${ }^{1}$, and Balpreet S. Ahluwalia ${ }^{1}$ \\ ${ }^{1}$ Department of Physics and Technology, UiT The Arctic University of Norway, NO-9037 \\ Tromsø, Norway \\ ${ }^{2}$ Department of Clinical Medicine, UiT The Arctic University of Norway, NO-9037 Troms $\varnothing$, \\ Norway
}

\begin{abstract}
In this work we have explored the live-cell friendly nanoscopy method Multiple Signal Classification Algorithm (MUSICAL) for multi-colour imaging of various organelles and sub-cellular structures in the cardiomyoblast cell line H2c9. We have tested MUSICAL for fast (up to $230 \mathrm{~Hz}$ ), multi-colour time-lapse sequences of various sub-cellular structures (mitochondria, endoplasmic reticulum, microtubules, endosomes and nuclei) in living cells using low excitation-light dose. Challenges and opportunities with applying MUSICAL for studying rapid sub-cellular dynamics are discussed.
\end{abstract}

Keywords: fluorescence nanoscopy, fluctuation imaging, live cells, cardiomyoblasts

\section{INTRODUCTION}

Structural components of living cells are challenging to study because of their size, sensitivity, and highly dynamic nature. Still, understanding the different cellular features and their interactions is crucial for comprehending basic cellular functions, and for finding potential treatments for cellular malfunction. Overcoming the challenges in monitoring the position and morphology of sub-cellular structures in living cells is hence of great interest. Fluorescence nanoscopy ${ }^{1}$ offers excellent contrast and specificity, but time and photon budget are limiting factors when resolution beyond the Abbe limit is required in live-cell imaging. Temporal resolution is limited and determined by the imaging system, technique and implementation, while the photon budget depends on the selected fluorophores, illumination mode and cellular system with varying label densities and chemical environments. Fluctuation-based computational nanoscopy techniques like Multiple Signal Classification Algorithm $(\text { MUSICAL) })^{2}$ extract super-resolved information from image sequences and are highly feasible for multi-colour experiments, contrasting localization-based nanoscopy in these regards. A caveat of time-accumulated live-cell images created from image sequences is, however, that (intra-)cellular dynamics smear structural details, compromising achievable resolution and the reliability of reconstructed features. The resulting artifacts can be difficult to distinguish from stationary biological components and therefore, imaging speed is critical for reliable image reconstruction. In this work, we have acquired fluctuation data using a commercial widefield system and sCMOS cameras in fast acquisition mode and tested the abilities and challenges of MUSICAL in capturing cellular details and dynamics in the rapidly changing microenvironment of living cardiomyoblasts.

\section{METHODS}

The cardiomyoblast cell line H9c2 was cultivated in glass bottom dishes and transiently transfected with organelle targeted fluorescent fusion proteins and labelled with live-cell compatible fluorescent labels, taking care to reduce label-induced toxicity. The cells were imaged at room temperature, but in heated media and for less than 30-60 min. Widefield, time-lapse data was acquired as fast as the system (OMX V4 optical microscope with 3 cameras and up to four-channel imaging) allowed while maintaining a signal-to-background ratio (SBR) of about 4 to 1.

\footnotetext{
*Correspondence: ida.s.opstad@uit.no
} 
For each colour channel various combinations of intensity and exposure time were acquired to find a good tradeoff between SBR, photo-bleaching and temporal resolution. MUSICAL images were first generated for a single time-point for various numbers of frames (10-400) and threshold values (based on image stack singular values) to select suitable reconstruction parameters before generating MUSCIAL time-lapses from raw, low intensity image sequences (up to 3000 frames per colour channel). The MUSICAL image reconstructions were generated using a new Python implementation of MUSICAL optimized for high-speed reconstruction, making it feasible to generate MUSICAL time-lapse images of large, multi-colour image stacks. ${ }^{3}$

\section{RESULTS AND DISCUSSION}

For the current state of MUSICAL, thresholds for reconstruction must be selected manually. We found that a suitable selection way to be such that image features appearing in-focus were emphasized and out-of-focus features were suppressed in the MUSICAL images. 50 frames were in most cases found to give a suitable compromise between motion-smearing and fluctuation data for the algorithm, though longer sequences often appeared to provide a more reliable reconstruction if the organelles remained reasonably stationary within the sequence. For time-lapse sequences where considerable dynamics occurred, the algorithm was found to give a very strong image intensity. On the one hand, structural motion creates strong image intensity fluctuations that out-compete the intrinsic intensity fluctuations of the fluorophores. On the other hand, the algorithm appears to provide a window into a new way of recognizing and analyzing sub-cellular dynamics, which is key to understanding living processes and systems. Figure 1 shows results applying the algorithm in fast-acquisition mode for one colour channel at a time for mitochondria (upper panels) and endoplasmic reticulum (ER; lower panels). Figure 2 displays results applying MUSICAL to four-colour time-lapse data of (b) endosomes, (c) mitochondria, (d) ER and (e) nucleus. Although no challenge for the algorithm in itself, four-colour acquisition was slow (0.49s per time-point) due to
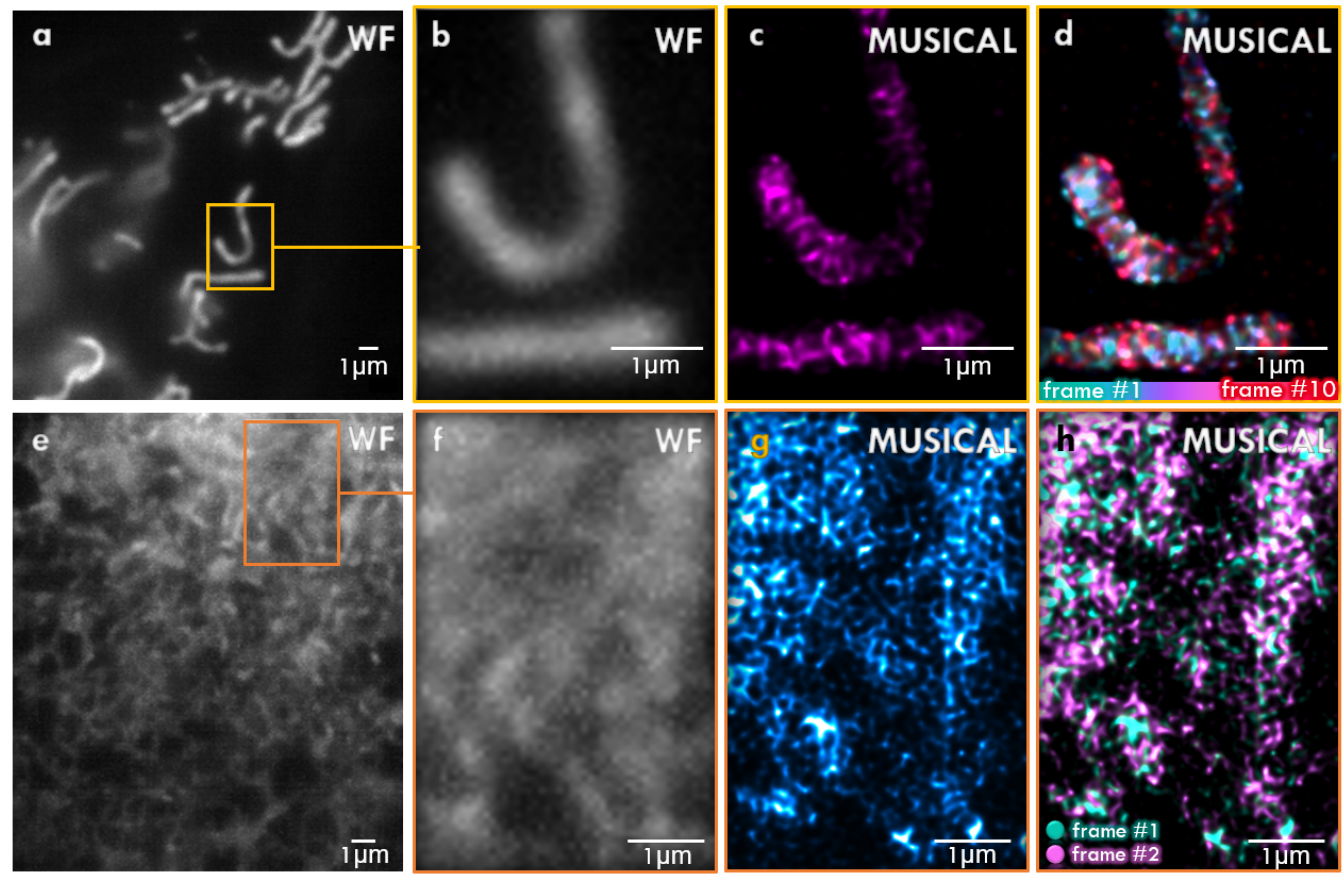

Figure 1. Pushing the limits of time resolution in live-cell nanoscopy. Obtainable MUSICAL frame rate is limited by achievable system imaging speed and the fluorescence intensity fluctuations of the raw time-lapse image data. Upper panels: mitochondrial inner membrane, lower panels: endoplasmic reticulum (ER). (a) and (e): widefield images (50 frames low intensity images maximum intensity projected) with indicated regions magnified on the right. (c) and (g) corresponding MUSICAL images obtained from the 50 frames projected on the left. (d) and (h) MUSICAL timelapse images colour projected as indicated in the colour bars. The different colour projected time-points are $0.22 \mathrm{~s}$ apart (acquisition time for a sequence of 50 widefield images). 

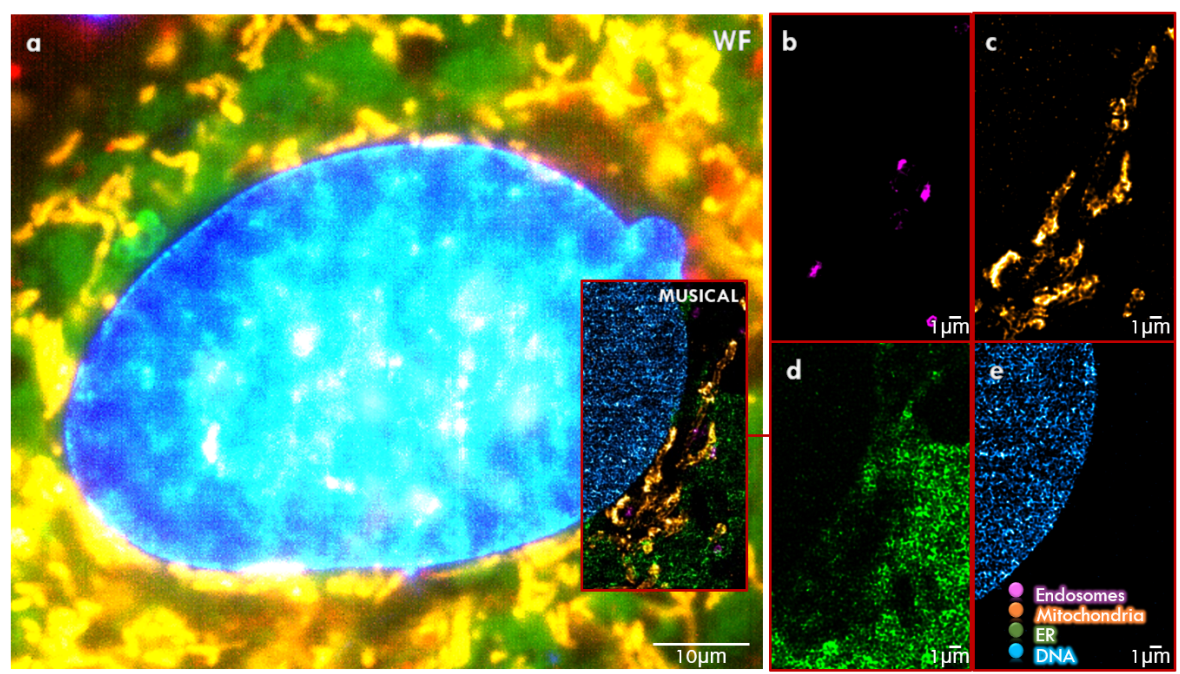

Figure 2. Multi-colour imaging with MUSICAL.(a) Single frame widefield image (raw MUSICAL data) of a living cardiomyoblast, labelled with four different fluorescent tags (acquisition time: 0.49s). The four-colour MUSICAL insert is shown to the right with separated channels. (b) Endosomes labelled using the membrane probe mCLING-ATTO647N, (c) mitochondrial inner membrane (dsRed-mito). (d) ER fusion protein eGFP-KDEL. (e) Nucleus labelled using Hoechs. The acquisition time for one four-colour MUSICAL image was 23s (obtained from 47 widefield images).

necessary switching of fluorescent filters between each time-point (number of channels $>$ number of cameras), translating into algorithmic challenges in reconstructing moving objects even for short sequences.

\section{CONCLUSION}

In conclusion, we have explored the computational nanoscopy approach MUSICAL in fast, multi-colour nanoscopy of living cardiomyoblasts. The algorithm is promising with its multi-colour abilities and live-cell compatibility. Challenges include recognition and elimination of reconstruction artefacts, fast three-dimensional motion of living cellular features and modest intrinsic fluorescent intensity fluctuations compared to motion-induced signal fluctuations. Although an analytical challenge, this latter feature of the algorithm can be exploited in analyzing feature dynamics of e.g. cellular organelles.

\section{ACKNOWLEDGMENTS}

Funding information

FS acknowledges funding from the European Molecular Biology Organisation (\#7411) and Marie SkłodowskaCurie actions (\#836355). KA acknowledges funding from Marie Skłodowska-Curie actions (SEP-210382872). BSA acknowledges funding from UiT, The Arctic Univeristy of Norway, Tematiske Satsinger program. ABB and TM acknowledges funding from the Health Authorities of North Norway.

\section{Competing interests statement}

The authors declare no competing interests.

\section{REFERENCES}

[1] Sahl, S. J., Hell, S. W., and Jakobs, S., "Fluorescence nanoscopy in cell biology," Nature reviews Molecular cell biology 18(11), 685 (2017).

[2] Agarwal, K. and Macháň, R., "Multiple signal classification algorithm for super-resolution fluorescence microscopy," Nature communications 7, 13752 (2016).

[3] Acuña-Maldonado, S. A., Multiple Signal Classification Algorithm: computational time reduction and pattern recognition applications, Master's thesis, UiT The Arctic University of Norway, Troms $\varnothing$, Norway (2019). 